nephron

Practice
Nephron 2017;135:287-290

DOI: $10.1159 / 000455130$
Received: August 1, 2016

Accepted after revision: December 12, 2016

Published online: February 4, 2017

\title{
In the Wake of Systolic Blood Pressure Intervention Trial: New Targets for Improving Hypertension Management in Chronic Kidney Disease?
}

\author{
Crystal C. Tyson ${ }^{a}$ Thomas M. Coffman ${ }^{a, b}$ \\ ${ }^{a}$ Division of Nephrology, Department of Medicine, Duke University Medical Center, Durham, NC, USA; ${ }^{b}$ Duke-National \\ University of Singapore (Duke-NUS) Medical School, Singapore, Singapore
}

\section{Keywords}

Hypertension · Kidney disease $\cdot$ Cardiovascular disease

\begin{abstract}
Systolic Blood Pressure Intervention Trial (SPRINT) was a multicenter randomized controlled trial showing the significant benefit of intensive reduction of blood pressure to a target of $120 \mathrm{~mm} \mathrm{Hg}$ in individuals with hypertension and elevated cardiovascular risk. Because SPRINT includes the largest cohort of adults with chronic kidney disease (CKD) to be prospectively studied in a hypertension intervention trial, it has particular relevance to the field of nephrology. Here, we review the findings of SPRINT and assess their potential impact on guidelines for treatment of hypertension in patients with CKD. We believe that the data from SPRINT will support a recommendation for lowering blood pressure targets to $120 \mathrm{~mm} \mathrm{Hg}$ in a substantial segment of adults with CKD.

(c) 2017 S. Karger AG, Basel
\end{abstract}

Hypertension is one of the most common disorders of humankind, and the kidney has a prominent role as both a cause and target of elevated blood pressure [1]. Proportional associations between blood pressure and cardiovascular risk [2], along with prospective benefits of lowering blood pressure to improve cardiovascular outcomes [3] have been well-established in epidemiological studies and clinical trials. Such observations have fueled decades of research focused on developing better approaches for preventing and treating hypertension. While much progress has been made over the years in identifying a range of effective blood pressure lowering therapies, a critical clinical question has persisted: "how low should blood pressure be reduced to provide optimal protection against end-organ damage?" A definitive answer to this question would have a profound impact on public health considering that millions of people worldwide die annually from heart disease, stroke, and kidney disease, for which hypertension is a major contributor [4].

The burden of hypertension and cardiovascular disease in chronic kidney disease (CKD) is particularly striking. Compared to the general population, hypertension is 2-3 times more prevalent in CKD [5]. Kidney disease is also independently associated with cardiovascular disease and death, with substantially higher risk occurring in more advanced stages of disease [6]. Furthermore, individuals with CKD are more likely to die from a cardiovascular event than to develop end-stage renal disease $[7,8]$. Therefore, reducing cardiovascular risk in CKD is arguably the major clinical challenge facing the field of nephrology today. In this regard, the Systolic Blood Pressure Intervention Trial (SPRINT), a landmark trial testing whether intensively lowering blood pressure improves cardiovascular outcomes, provides important new findings relevant to individuals with kidney disease [9]. 
SPRINT was a randomized, controlled trial testing whether reducing the systolic blood pressure (SBP) to a target of $120 \mathrm{~mm} \mathrm{Hg}$ would diminish cardiovascular events compared to the accepted standard of $140 \mathrm{~mm} \mathrm{Hg}$ [9]. The trial enrolled a total of 9,361 adults aged $\geq 50$ years with SBP $\geq 130 \mathrm{~mm} \mathrm{Hg}$ and evidence of increased cardiovascular risk. While SPRINT was primarily designed to define blood pressure targets in adults at risk for cardiovascular events, 2,646 adults with CKD were enrolled, comprising $28 \%$ of the entire cohort and exceeding the number of combined participants enrolled in the 3 previous major trials designed to evaluate different blood pressure targets on kidney function in patients with CKD: Modification of Diet in Renal Disease [10], African American Study of Kidney Disease and Hypertension [11], and Ramipril Efficacy In Nephropathy [12]. Thus, SPRINT comprises the largest prospective cohort of individuals with CKD studied in a randomized, prospective trial testing optimal levels of blood pressure control.

In SPRINT, separation of blood pressure was successfully achieved between the treatment groups. Mean SBP in the intensive treatment group was reduced by $15 \mathrm{~mm}$ $\mathrm{Hg}$, compared to the standard therapy (121 vs. $136 \mathrm{~mm}$ $\mathrm{Hg}$ ). Remarkably, the mean number of anti-hypertensive medications used to achieve this separation was only 2.8 in the intensive group compared to 1.8 in the standard group. The primary outcome in SPRINT was a composite of myocardial infarction, acute coronary syndrome, stroke, decompensated heart failure, and cardiovascularrelated death. The study was terminated prematurely after the data and safety monitoring board found evidence of benefit in the group subjected to intensive treatment. Specifically, the event rate of the primary composite outcome was significantly lower for those who received intensive therapy ( $1.65 \%$ per year) compared to standard therapy (2.19\% per year), representing a $25 \%$ reduction in relative risk. Furthermore, these results were consistent across pre-specified clinical subgroups including age, sex, race, tertiles of SBP, history of cardiovascular disease, and importantly in the context of this review, history of CKD. With the stipulation that some CKD subgroups were excluded from participation, including individuals with estimated glomerular filtration rate (eGFR) $<20 \mathrm{~mL} /$ $\mathrm{min} / 1.73 \mathrm{~m}^{2}$, proteinuria $\geq 1 \mathrm{~g} /$ day, diabetes, and polycystic kidney disease, findings from SPRINT indicate that intensive blood pressure lowering can improve cardiovascular outcomes for individuals with CKD.

Previous observational studies suggested the existence of a so-called "U-curve" relationship between blood pressure and $\mathrm{CV}$ risk across populations of individuals with
$\mathrm{CKD}$, where risk was paradoxically increased in individuals with lower blood pressure ranges [13-15]. Such studies had raised concerns that lowering the blood pressure even below $140 \mathrm{~mm} \mathrm{Hg}$ might actually cause harm in patients with kidney disease. However, causal relationships cannot be established through observational studies and it was suggested that low blood pressure might actually be a surrogate for failing health or other comorbidities impacting risk. SPRINT now provides level 1 evidence indicating that aggressive lowering of blood pressure improves cardiovascular risk in a selected population with CKD.

It should be noted that blood pressure measurements in SPRINT were carried out using an automated measurement system after the patient had been seated quietly and unattended for $5 \mathrm{~min}$ [16]. The aim of this approach was to achieve more precise measurements, avoiding the potentially confounding issue of "white coat" hypertension, but with the proviso that blood pressure values obtained through this method may be systematically lower than conventional office measurements. Thus, while SPRINT has clearly demonstrated the benefits of intensive blood pressure lowering, questions have been raised about translation of data from the clinical trial into specific numerical targets to be safely implemented in the real world [17]. This issue will likely be clarified as more data emerge from SPRINT, including information on subsets of participants who underwent ambulatory blood pressure monitoring. On the other hand, it could be argued that measurement with automated devices after resting should be adopted as the standard method for determining blood pressure in the outpatient setting.

Pre-specified secondary kidney outcomes were also assessed in SPRINT. Among participants with CKD at baseline, there was no significant difference between the intensive and standard treatment groups in the relatively small number of participants with a decline in eGFR of $\geq 50 \%$ ( 0.8 vs. $0.8 \%$ ), incident albuminuria (9.3 vs. $11.8 \%$ ), incident end-stage renal disease requiring dialysis ( $0.5 \mathrm{vs.}$ $0.8 \%$ ), incident kidney transplant ( 0 vs. $0 \%$ ), or a composite of each of these outcomes (1.1 vs. 1.1\%). A caveat here is that the number of kidney outcomes in SPRINT was relatively small, likely because individuals with proteinuria, a major risk factor for progressive CKD, were excluded from the trial. Thus, in patients with CKD, there was no detectable benefit of intensive blood pressure lowering on CKD progression, but also no evidence of harm.

In contrast, for the subgroup with normal kidney function, intensive therapy was associated with a significantly higher incidence of eGFR decline, defined as $\geq 30 \%$ reduction in eGFR to a value $<60 \mathrm{~mL} / \mathrm{min} / 1.73 \mathrm{~m}^{2}$ (3.8 vs. $1.1 \%$ 
with standard therapy). Irrespective of CKD status, intensive therapy was also associated with a significantly higher incidence of acute kidney injury compared to standard therapy ( 4.4 vs. $2.6 \%, p<0.001$ ). The mechanisms underlying this preponderance of adverse kidney events are not clear. In SPRINT, the standard for assessing deterioration in kidney function among individuals without CKD was a $30 \%$ decline in eGFR, compared to $50 \%$ in individuals with CKD. This less stringent threshold may account for the higher rate of events observed for this subgroup. Furthermore, renin-angiotensin-aldosterone inhibitors were used more frequently in the intensive therapy group, which may have contributed to the overall decline in eGFR as well as the higher incidence of acute kidney injury. Despite these adverse kidney events, intensive therapy reduced the cardiovascular end points for individuals whether CKD was present or not (hazard ratio 0.7 and 0.8 , respectively; $p$ for interaction $=0.36$ ), suggesting that the benefits of intensive therapy may outweigh the risks. Nonetheless, additional time and follow-up will be required to determine whether there will be longer term impact on kidney health and mortality.

SPRINT clearly provides strong evidence that lowering SBP to a goal of $120 \mathrm{~mm} \mathrm{Hg}$ compared to current targets of $140 \mathrm{~mm} \mathrm{Hg}$ improves cardiovascular outcomes in adults with increased cardiovascular risk. This also seems to hold true for individuals with CKD. However, since diabetics were excluded from SPRINT, the trial does not provide guidance on optimal targets for blood pressure in diabetes, a powerful cardiovascular risk factor and major cause of progressive CKD. This issue was addressed in a previous trial, ACCORD, a prospective randomized clinical trial with many similarities to SPRINT, testing in diabetics whether lowering blood pressure to $120 \mathrm{~mm} \mathrm{Hg}$ compared to $140 \mathrm{~mm} \mathrm{Hg}$ would improve cardiovascular outcomes [18]. Although a significant difference in lowering of blood pressure was achieved in the intensively treated group with a magnitude very similar to SPRINT $(15 \mathrm{~mm}$ $\mathrm{Hg}$ reduction in SBP), there was no significant benefit in cardiovascular outcomes. While eGFR was lower with intensivebloodpressurelowering $\left(75 \mathrm{vs} .81 \mathrm{~mL} / \mathrm{min} / 1.73 \mathrm{~m}^{2}\right.$; $p<0.001)$, frequency of macroalbuminuria was reduced (6.6 vs. $8.7 \%, p=0.009$ ), but, as in SPRINT, there was no beneficial effect on the progression to end-stage renal disease ( 2.5 vs. $2.4 \%$ ). In retrospect, because of an unexpectedly low frequency of cardiovascular events in ACCORD, it has been suggested that it was underpowered, with 4,733 participants compared to 9,361 in SPRINT. Nonetheless, at this point, there is no firm evidence supporting blood pressure targets below current recommendations of
140/90 $\mathrm{mm} \mathrm{Hg}$ for persons with diabetes in reducing cardiovascular risk or kidney disease progression.

In summary, SPRINT is truly a landmark trial in hypertension therapy that will surely impact blood pressure guidelines, suggesting an optimal target of $120 \mathrm{~mm} \mathrm{Hg}$ for therapy in adults with hypertension and increased risk for cardiovascular disease. The findings from SPRINT suggest this target should also apply to individuals with CKD, with the exception of those with proteinuria, diabetes, or polycystic kidney disease [19] where benefits of more intensive blood pressure lowering have not yet been clearly established. While blood pressure reduction in SPRINT reduced the cardiovascular risk in $\mathrm{CKD}$, there was no significant effect on kidney disease progression albeit in a cohort with low rates of kidney outcomes. The intensive blood pressure target was achieved with, on average, one additional anti-hypertensive drug, suggesting that achieving the lower goal is feasible without undue patient or financial burden. However, this was at the cost of some adverse effects including higher rates of acute kidney injury for all participants and greater eGFR decline in participants who entered the trial with normal kidney function. The long-term consequences of these apparent adverse kidney effects are not yet clear. Certainly the risks and benefits of intensive blood pressure reduction will be further clarified as additional analyses of the SPRINT data come to light. If intensive therapy is implemented, clinicians should monitor patients carefully and be prepared to de-escalate treatment in the event of symptomatic hypotension or other adverse events. But for now, the findings from SPRINT suggest that intensive blood pressure reduction to a systolic goal of $120 \mathrm{~mm} \mathrm{Hg}$ will attenuate cardiovascular risk in a large segment of patients with CKD.

\section{Disclosure Statement}

The authors have no conflicts of interest to declare.

$\begin{aligned} \text { References } & \text { Coffman TM: The inextricable role of the kid- } \\ & \text { ney in hypertension. J Clin Invest 2014;124: } \\ & 2341-2347 . \\ 2 & \text { Kannel WB: Elevated systolic blood pressure } \\ & \text { as a cardiovascular risk factor. Am J Cardiol } \\ & 2000 ; 85: 251-255 . \\ 3 & \text { Lewington S, Clarke R, Qizilbash N, Peto R, } \\ & \text { Collins R; Prospective Studies Collaboration: } \\ & \text { Age-specific relevance of usual blood pres- } \\ & \text { sure to vascular mortality: a meta-analysis of } \\ & \text { individual data for one million adults in 61 } \\ & \text { prospective studies. Lancet 2002;360:1903- } \\ & 1913 .\end{aligned}$


4 World Health Organization: Global Health Estimates: Deaths by Cause, Age, Sex and Country, 2000-2012. Geneva, WHO, 2014.

5 United States Renal Data System: 2015 USRDS Annual Data Report: Epidemiology of Kidney Disease in the United States. Bethesda, National Institutes of Health, National Institute of Diabetes and Digestive and Kidney Diseases, 2015.

-6 Go AS, Chertow GM, Fan D, McCulloch CE, Hsu CY: Chronic kidney disease and the risks of death, cardiovascular events, and hospitalization. N Engl J Med 2004;351:1296-1305.

-7 Foley RN, Murray AM, Li S, Herzog CA, McBean AM, Eggers PW, Collins AJ: Chronic kidney disease and the risk for cardiovascular disease, renal replacement, and death in the United States Medicare population, 1998 to 1999. J Am Soc Nephrol 2005;16:489-495.

8 Keith DS, Nichols GA, Gullion CM, Brown JB, Smith DH: Longitudinal follow-up and outcomes among a population with chronic kidney disease in a large managed care organization. Arch Intern Med 2004;164:659-663.

-9 SPRINT Research Group, Wright JT Jr, Williamson JD, Whelton PK, Snyder JK, et al: A randomized trial of intensive versus standard blood-pressure control. N Engl J Med 2015; 373:2103-2116.

10 Klahr S, Levey AS, Beck GJ, Caggiula AW, Hunsicker L, Kusek JW, Striker G: The effects of dietary protein restriction and blood-pressure control on the progression of chronic renal disease. Modification of Diet in Renal Dis- ease Study group. N Engl J Med 1994;330: 877-884.

11 Wright JT Jr, Bakris G, Greene T, Agodoa LY, Appel LJ, Charleston J, Cheek D, DouglasBaltimore JG, Gassman J, Glassock R, Hebert L, Jamerson K, Lewis J, Phillips RA, Toto RD, Middleton JP, Rostand SG; African American Study of Kidney Disease and Hypertension Study Group: Effect of blood pressure lowering and antihypertensive drug class on progression of hypertensive kidney disease: results from the AASK trial. JAMA 2002;288: 2421-2431.

12 Ruggenenti P, Perna A, Loriga G, Ganeva M, Ene-Iordache B, Turturro M, Lesti M, Perticucci E, Chakarski IN, Leonardis D, Garini G, Sessa A, Basile C, Alpa M, Scanziani R, Sorba G, Zoccali C, Remuzzi G; REIN-2 Study Group: Blood-pressure control for renoprotection in patients with non-diabetic chronic renal disease (REIN-2): multicentre, randomised controlled trial. Lancet 2005;365: 939-946.

13 Messerli FH, Panjrath GS: The J-curve between blood pressure and coronary artery disease or essential hypertension: exactly how essential? J Am Coll Cardiol 2009;54:18271834.

14 Kovesdy CP, Bleyer AJ, Molnar MZ, Ma JZ, Sim JJ, Cushman WC, Quarles LD, KalantarZadeh K: Blood pressure and mortality in U.S. veterans with chronic kidney disease: a cohort study. Ann Intern Med 2013;159:233242.
15 Weiss JW, Peters D, Yang X, Petrik A, Smith DH, Johnson ES, Thorp ML, Morris C, O'Hare AM: Systolic BP and mortality in older adults with CKD. Clin J Am Soc Nephrol 2015;10:1553-1559.

-16 Cushman WC, Whelton PK, Fine LJ, Wright JT Jr, Reboussin DM, Johnson KC, Oparil S; SPRINT Study Research Group: SPRINT trial results: latest news in hypertension management. Hypertension 2016;67:263-265.

17 Kjeldsen SE, Lund-Johansen P, Nilsson PM, Mancia G: Unattended blood pressure measurements in the systolic blood pressure intervention trial: implications for entry and achieved blood pressure values compared with other trials. Hypertension 2016;67:808812

18 ACCORD Study Group, Cushman WC, Evans GW, Byington RP, Goff DC Jr, Grimm RH Jr, Cutler JA, Simons-Morton DG, Basile JN, Corson MA, Probstfield JL, Katz L, Peterson KA, Friedewald WT, Buse JB, Bigger JT, Gerstein HC, Ismail-Beigi F: Effects of intensive blood-pressure control in type 2 diabetes mellitus. N Engl J Med 2010;362:1575-1585.

19 Schrier RW, Abebe KZ, Perrone RD, Torres VE, Braun WE, Steinman TI, Winklhofer FT, Brosnahan G, Czarnecki PG, Hogan MC, Miskulin DC, Rahbari-Oskoui FF, Grantham JJ, Harris PC, Flessner MF, Bae KT, Moore CG, Chapman AB; HALT-PKD Trial Investigators: Blood pressure in early autosomal dominant polycystic kidney disease. N Engl J Med 2014;371:2255-2266. 